\title{
The Detrimental Role Played by Lipocalin-2 in Alcoholic Fatty Liver in Mice
}

Yan Cai, ${ }^{*}$ Alvin Jogasuria, ${ }^{\dagger}$ Huquan Yin, ${ }^{\ddagger}$ Ming-Jiang $\mathrm{Xu}^{*}{ }^{*}$ Xudong $\mathrm{Hu}^{\dagger \S}$ Jiayou Wang, ${ }^{\dagger \uparrow}$ Chunki Kim, ${ }^{\dagger}$ Jiashin Wu, ${ }^{\dagger}$ Kwangwon Lee, ${ }^{\dagger}$ Bin Gao, ${ }^{*}$ and Min You

\begin{abstract}
From the Laboratory of Liver Diseases, * National Institute on Alcohol Abuse and Alcoholism, NIH, Bethesda, Maryland; the Department of Pharmaceutical Sciences, ${ }^{\dagger}$ College of Pharmacy, Northeast Ohio Medical University, Rootstown, Ohio; the Department of Molecular Pharmacology and Physiology, ${ }^{\ddagger}$ University of South Florida Health Sciences Center, Tampa, Florida; the Department of Biology, ${ }^{\S}$ School of Basic Medical Science, Shanghai University of Traditional Chinese Medicine, Shanghai, China; and the Department of Anatomy, ${ }^{\top}$ School of Fundamental Medical Science, Guangzhou University of Chinese Medicine, Guangzhou, Guangdong, China
\end{abstract}

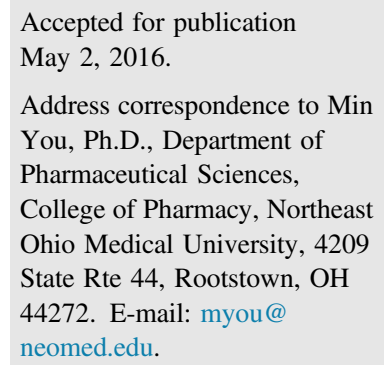

\begin{abstract}
We have previously shown that the ethanol-mediated elevation of lipocaline-2 (LCN2) is closely associated with the development of alcoholic fatty liver disease (AFLD) in mice. Herein, we aimed to understand the functional significance of LCN2 induction by ethanol and to explore its underlying mechanisms. We evaluated the effects of LCN2 in an in vitro cellular alcoholic steatosis model and in an animal study using wild-type and LCN2 knockout mice fed for 4 weeks with an ethanol-supplemented Lieber-DeCarli diet. In the cellular model of alcoholic steatosis, recombinant LCN2 or overexpression of LCN2 exacerbated ethanol-induced fat accumulation, whereas knocking down LCN2 prevented steatosis in hepatocytes exposed to ethanol. Consistently, removal of LCN2 partially but significantly alleviated alcoholic fatty liver injury in mice. Mechanistically, LCN2 mediates detrimental effects of ethanol in the liver via disrupted multiple signaling pathways, including aberrant nicotinamide phosphoribosyltransferase-sirtuin 1 axis, perturbed endocrine metabolic regulatory fibroblast growth factor 15/19 signaling, and impaired chaperone-mediated autophagy. Finally, compared with healthy human livers, liver samples from patients with AFLD had lower gene expression of several LCN2-regualted molecules. Our study demonstrated a pivotal and causal role of LCN2 in the development of AFLD and suggested that targeting the LCN2 could be of great value for the treatment of human AFLD. (Am J Pathol 2016, 186: 2417-2428; http://dx.doi.org/10.1016/j.ajpath.2016.05.006)
\end{abstract}

Alcoholic fatty liver disease (AFLD) encompasses a spectrum of liver histology, from steatosis to steatohepatitis, which can further progress to fibrosis, cirrhosis, liver cancer, and liver failure in clinical alcoholics. ${ }^{1}$ Because of the complex pathophysiology of AFLD, the exact underlying mechanisms are far from understood.

Lipocaline-2 (LCN2) [alias $24 \mathrm{p} 3$ protein in mouse, and neutrophil gelatinase-associated lipocalin (NGAL) in human, $\alpha 1$-microglobulin-related protein, or uterocalin] is a $25-\mathrm{kDa}$ secretory glycoprotein. ${ }^{2}$ LCN2 is abundantly present in the circulation. ${ }^{2,3}$ Although originally identified in human neutrophils, LCN2 is also expressed in various tissues, including liver, adipose, brain, spleen, lung, kidney, and intestine. ${ }^{2,3}$ One of the LCN2 receptors, LCN2R (24p3R in mouse or NGALR2/SLC22A17 in humans), is a membrane-associated protein. ${ }^{4}$ 24p3R expressing mammalian cells are sensitive to LCN2-mediated signals, such as apoptosis through modulation of iron metabolism. ${ }^{4}$

LCN2 expression and production can be induced under various pathophysiological conditions, such as infection, inflammation, burn injury, kidney and cardiovascular diseases, and intoxication, and thus, serve as a reliable biomarker of these diseases. ${ }^{2,5}$ At the molecular level, LCN2 expression and production are highly regulated by metabolic stress signals, such as lipopolysaccharide (LPS), proinflammatory

Supported by National Institute on Alcoholism and Alcohol Abuse grants AA013623 and AA015951 (M.Y.).

C.Y. and A.J. contributed equally to this work.

Disclosures: None declared. 
cytokines, reactive oxygen species-producing agents, dexamethasone, and glucocorticoids. ${ }^{2,3,6}$

Although it has been well established as an important regulator of iron metabolism, $\mathrm{LCN} 2$ has recently emerged as a pivotal modulator of lipid metabolism and inflammation, ${ }^{2,3}$ and has been suggested to serve complex and controversial functional roles in the pathogenesis of liver diseases. ${ }^{7-17}$ It was reported that LCN2 gene and protein expression levels were significantly elevated in the livers of $d b / d b$ mice. ${ }^{15}$ Serum LCN2 concentration was elevated in diabetic patients and hepatic LCN2 level was closely associated with nonalcoholic fatty liver disease in severely obese women. ${ }^{15,16}$ Experimentally induced acute liver injuries, such as bile duct ligation and repeated application of carbon tetrachloride, rapidly and markedly elevated LCN2. ${ }^{7-14}$ Intriguingly, LCN2 deficiency mice displayed more carbon tetrachlorideinduced liver damage, accompanied by higher expressions of inflammatory cytokines and chemokines, such as IL-1 $\beta$, IL-6, tumor necrosis factor- $\alpha$, and monocyte chemoattractant protein-1/the chemokine (C-C motif) ligand 22 in hepatocytes, suggesting that liver injury-induced up-regulation of LCN2 might have hepatoprotective effects against liver injuries. ${ }^{17}$ Moreover, hepatocytes were responsible for LCN2 production after bacterial infection or partial hepatectomy, and hepatocyte-derived LCN2 played a protective role in inhibiting bacterial infection and promoting liver regeneration. ${ }^{13}$

Hepatic and serum LCN2 levels were drastically elevated in response to chronic or chronic-binge ethanol administration and were closely linked with the development and progression of alcohol-induced fatty liver injury in these mice. ${ }^{18-21}$ Nevertheless, the causes of LCN2 induction in response to ethanol exposure and the causal role of LCN2 in the development of early stage alcoholic liver disease, alcoholic steatosis, are still unknown. The present study investigated the functional significance of the LCN2 induction in response to alcohol exposure using in vitro cell culture and in vivo animal model of AFLD, and in human AFLD samples. We also explored the mechanisms of LCN2 induction in response to ethanol challenge.

\section{Materials and Methods}

\section{Plasmids, Antibodies, and Reagents}

Murine Ad plasmids (Ad-GFP, Ad-LCN2, Ad-SIRT1wt, AdsiRNAcontrol, Ad-LCN2siRNA Ad-SIRT1wt, Ad-SIRT1siRNA) were custom designed and obtained through using Custom Adenovirus Construction Service from Vector Biolabs (Malvern, PA). miRIDIAN miR-217 mimic (miR-217) and miRIDIAN hairpin inhibitor miR-217 (anti-miR-217) were purchased from Thermo Scientific (Middletown, VA). Plasmids for control vector, wild-type SIRT1 (SIRT1wt), and pGL3-24p3/LCN2-Luciferase reporter (25463) were purchased from Addgene Inc. (Cambridge, MA). SIRT1, LC3A/B, and glyceraldehyde-3-phosphate dehydrogenase antibodies were purchased from Cell Signaling Technology (Danvers, MA). LCN2 and Nampt antibodies were purchased from Abcam (Cambridge, UK). P62 antibody was purchased from Sigma (St. Louis, MO). LPS (tissue culture-tested, L-2654) and ethanol, 4-methylpyrazole, and cyanamide were purchased from Sigma Chemical (St. Louis, MO). Proteasome inhibitor MG132 was purchased from EMD Chemicals (Gibbstown, NJ). Cyclosporine A was purchased from Novartis (Basel, Switzerland). Most chemicals and supplies were purchased from Sigma Chemical (St. Louis, MO), Schleicher and Schuell (Keene, NH), GIBCO-BRL (Grand Island, NY), and DuPont NEN Research Products (Boston, MA). Recombinant mouse LCN2 was purchased from Cell Signaling Technology (Danvers, MA).

\section{Animals}

All mice were maintained on a normal chow diet (NIH-31 Open Formula Mouse/Rat Sterilizable Diet 7917; Teklad Diets, Madison, WI) before beginning the ethanol feeding study. Global LCN2 knockout (Lcn2KO) mice were kindly provided by Dr. Tak Mak (University of Toronto, Toronto, Canada) and $L c n 2^{\text {flox/flox }}$ mice were generated and described in detail previously. ${ }^{13}$ Hepatocyte-specific Lcn 2 knockout (Lcn2LKO) mice were generated by crossing albumin-Cre mice with $L c n 2^{\text {flox/flox }}{ }^{13}$ Both $L c n 2 \mathrm{KO}$ and Lcn2LKO mice were backcrossed to a C57BL/6N background in the National Institute on Alcohol Abuse and Alcoholism animal facility. C57BL/6N mice were used as wild-type (WT) controls for $L c n 2 \mathrm{KO}$ mice. Littermate Cre-negative floxed mice were used as WT controls for Lcn2LKO mice.

\section{Diet Formulation and Chronic Ethanol Feeding Studies}

Lieber-DeCarli control and ethanol liquid diets were purchased from Bio-Serv (Frenchtown, NJ). ${ }^{22}$ The nutrient profile of each diet was identical, excluding carbohydrate content. Ethanol and control diets both contained $15.1 \%$ calories from protein and $35.9 \%$ calories from fat. Control diet contained $49 \%$ calories from carbohydrates, whereas there were $20.6 \%$ calories from carbohydrates in the ethanol diet. The remaining $28.4 \%$ of calories in the ethanol diet was from alcohol.

The 10- to 12-week-old male Lcn2KO mice and their agematched littermate (WT) controls were divided into following four dietary groups: WT control; WT plus ethanol (identical to the control diet but with $5 \% \mathrm{w} / \mathrm{v}$ ethanol added); Lcn $2 \mathrm{KO}$ control; and $L c n 2 \mathrm{KO}$ plus ethanol. ${ }^{19,22}$ All liquid diets were freshly prepared from powder, and the dietary and nutritional intake of control mice were matched to those of the ethanol-fed mice by pair feeding the same volume of isocaloric liquid diet for 4 weeks. Ethanol was introduced gradually into the diet as described previously. ${ }^{22}$ Food intake was recorded daily. After 4 weeks on the liquid diets, the mice were euthanized, and blood and tissue samples were collected. Using the same ethanol pair feeding 
Liber-DeCarli protocol, 10- to 12-week-old male Lcn2LKO and Littermate Cre-negative floxed (WT) mice were pair-fed with ethanol or control diets for 4 weeks. All in vivo animal protocols were approved by the National Institute on Alcohol Abuse and Alcoholism animal care and use committee and Institutional Animal Care and Use Committee of Northeast Ohio Medical University.

\section{Measurement of Liver Lipid and Hematoxylin and Eosin Staining}

Liver triglyceride cholesterol was measured as described previously. ${ }^{19}$ Liver tissues were fixed in $10 \%$ formalin and embedded in paraffin. Embedded liver tissues were cut into sections and used to stain with hematoxylin and eosin. ${ }^{19}$

\section{Staining for the $\mathrm{F} 4 / 80^{+}$and $\mathrm{MPO}$}

Embedded liver tissues were subjected to immunohistochemical staining for $\mathrm{F} 4 / 80^{+}$or myeloperoxidase (MPO) by using allophycocyanin (APC)-Alexa Fluor 750 conjugatedrat anti-mouse $\mathrm{F} 4 / 80^{+}$monoclonal antibody (Thermo Fisher Scientific, Rockford, IL) or rabbit anti-MPO polyclonal antibody (Abcam Inc., Cambridge, MA). Sections were incubated with Alexa Fluor 488 conjugated-goat anti-rabbit IgG secondary antibody (Thermo Fisher Scientific, Rockford, IL) for a MPO antibody. Positive cells for $\mathrm{F} 4 / 80^{+}$or MPO were randomly photographed using a fluorescence microscope, and positive density was quantified using Image-Pro Plus software version 5 (Media Cybernetics, Bethesda, MD).

\section{Enzyme-Linked Immunosorbent Assays from Mouse Serum}

Serum level of LCN2 was measured by Lipocalin-2/NGAL Mouse ELISA Kit (Abcam, Cambridge, MA). Serum level of SAA1 was measured by SAA1/ab157723 Mouse Elisa Kit (Abcam). Serum levels of alanine aminotransferase (ALT) and aspartate aminotransferase (AST) were determined using ALT/AST Infinity Kits (Thermo Scientific, Middletown, VA). Plasma or tissue fatty acids, triglyceride, and cholesterol were determined using the Sigma Diagnostics Triglyceride and Infinity Cholesterol Reagent. Analyses were performed with a Synergy HT microplate reader (BioTek, Winooski, VT).

\section{RNA Isolation and mRNA Level Analysis}

Total RNA was prepared from cells or mouse livers using TRIzol Reagent (Invitrogen, Carlsbad, CA), as described previously. ${ }^{19}$ Real-time quantitative PCR (qPCR) amplification was performed using the CFX96 Touch Real-Time PCR Detection System (Bio-Rad Laboratories, Hercules, CA) with FastStart Universal SYBR Green Master Mix with ROX (Roche Diagnostics, Indianapolis, IN). Gene-specific primer sets were obtained from Harvard's PrimerBank (http://pga.mgh.harvard.edu/primerbank; last accessed March 29, 2016) and purchased from QIAGEN SuperArray Bioscience (Frederick, MD). Primer sets were either designed or purchased (Table 1). The relative amount of target mRNA was calculated using the comparative cycle

Table 1 Primers Used in This Study

\begin{tabular}{|c|c|c|}
\hline Primer & Direction & Sequence \\
\hline \multirow[t]{2}{*}{$24 p 3 R$} & Fwd & 5'-TTTGGCCGTCGTGGGATTG-3' \\
\hline & Rev & $5^{\prime}-$ GGCGCATCAGGTAGACACC $-3^{\prime}$ \\
\hline \multirow[t]{2}{*}{$\beta-K L$} & Fwd & 5'-TGTTCTGCTGCGAGCTGTTAC- $3^{\prime}$ \\
\hline & $\operatorname{Rev}$ & 5'-CCGGACTCACGTACTGTTTTT-3' \\
\hline \multirow[t]{2}{*}{ CPT-1a } & Fwd & 5'-GCTTGTCTCAAGTGCTTCCC-3' \\
\hline & $\operatorname{Rev}$ & 5'-CCAGGCTACAGTGGGACATT-3' \\
\hline \multirow[t]{2}{*}{ CYP7A1 } & Fwd & 5'-TGTGCTTCAAGCTCTCAGCCAGC-3' \\
\hline & $\operatorname{Rev}$ & 5'-ACTAGTGGCGGGCGTCCCTA-3' \\
\hline \multirow[t]{2}{*}{ FGFR4 } & Fwd & 5'-GCTCGGAGGTAGAGGTCTTGT-3' \\
\hline & $\operatorname{Rev}$ & 5'-CCACGCTGACTGGTAGGAA-3' \\
\hline \multirow[t]{2}{*}{ FXR- $\alpha$} & Fwd & 5'-GGCAGAATCTGGATTTGGAATCG-3' \\
\hline & $\operatorname{Rev}$ & 5'-GCTGAACTTGAGGAAACGGG-3' \\
\hline \multirow[t]{2}{*}{ FXR- $\beta$} & Fwd & 5'-ATGCAGTTTCAGGGCTTAGAA-3' \\
\hline & $\operatorname{Rev}$ & 5'-CGGGACATTGTTGTATGGG-3' \\
\hline \multirow[t]{2}{*}{ GAPDH } & Fwd & 5'-TGACCTCAACTACATGGTCTACA-3' \\
\hline & $\operatorname{Rev}$ & 5'-СTTCCСАTTCTCGGCCTTG-3' \\
\hline \multirow[t]{2}{*}{ HSP70 } & Fwd & 5'-GAGATCGACTCTCTGTTCGAGG-3' \\
\hline & $\operatorname{Rev}$ & 5'-GCCCGTTGAAGAAGTCCTG-3' \\
\hline \multirow[t]{2}{*}{ HSP90aa1 } & Fwd & 5'-AATTGCCCAGTTAATGTCCTTGA-3' \\
\hline & $\operatorname{Rev}$ & 5'-CGTCCGATGAATTGGAGATGAG-3' \\
\hline \multirow[t]{2}{*}{ HSPA8 } & Fwd & 5'-TCTCGGCACCACCTACTCC-3' \\
\hline & $\operatorname{Rev}$ & 5'-CTACGCCCGATCAGACGTTT-3' \\
\hline \multirow[t]{2}{*}{ IL-1 $\beta$} & Fwd & 5'-TCGCTCAGGGTCACAAGAAA- 3' \\
\hline & $\operatorname{Rev}$ & $5^{\prime}$-CATCAGAGGCAAGGAGGAAAAC- $3^{\prime}$ \\
\hline \multirow[t]{2}{*}{ IL-6 } & Fwd & 5'-TAGTCCTTCCTACCCCAATTTCC-3' \\
\hline & $\operatorname{Rev}$ & 5'-TTGGTCCTTAGCCACTCСTTC-3' \\
\hline \multirow[t]{2}{*}{ LAMP-2A } & Fwd & 5'-TGTATTTGGCTAATGGCTCAGC- $3^{\prime}$ \\
\hline & $\operatorname{Rev}$ & 5'-TATGGGCACAAGGAAGTTGTC-3' \\
\hline \multirow[t]{2}{*}{ LCN2 } & Fwd & 5'-TGGCCCTGAGTGTCATGTG-3' \\
\hline & $\operatorname{Rev}$ & 5'-CTCTTGTAGCTCATAGATGGTGC-3' \\
\hline \multirow[t]{2}{*}{ MAP1LC3B } & Fwd & $5^{\prime}-$ AAGCAGCTGCCCGTCCTGGA-3' \\
\hline & $\operatorname{Rev}$ & 5'-TTGAGCTGCAAGCGCCGTCT-3' \\
\hline \multirow[t]{2}{*}{ MCP-1 } & Fwd & 5'-TTAAAAACCTGGATCGGAACCAA-3' \\
\hline & $\operatorname{Rev}$ & 5'-GCATTAGCTTCAGATTTACGGGT-3' \\
\hline \multirow[t]{2}{*}{ MIP-1 $\alpha$} & Fwd & 5'-TTCTCTGTACCATGACACTCTGC-3' \\
\hline & $\operatorname{Rev}$ & 5'-CGTGGAATCTTCCGGCTGTAG-3' \\
\hline \multirow[t]{2}{*}{ P62 } & Fwd & 5'-AGGATGGGGACTTGGTTGC-3' \\
\hline & $\operatorname{Rev}$ & 5'-TCACAGATCACATTGGGGTGC-3' \\
\hline \multirow[t]{2}{*}{ PGC- $1 \alpha$} & Fwd & 5'-TATGGAGTGACATAGAGTGTGCT-3' \\
\hline & $\operatorname{Rev}$ & $5^{\prime}-\mathrm{CCACTTCAATCCACCCAGAAAG-3^{ \prime }}$ \\
\hline \multirow[t]{2}{*}{ PPAR $\alpha$} & Fwd & 5'-TTTCGGCGAACTATTCGGCTG-3' \\
\hline & $\operatorname{Rev}$ & 5'-GGCATTTGTTCCGGTTCTTCTT-3' \\
\hline \multirow[t]{2}{*}{ SAA-1 } & Fwd & 5'-TTAGCTCAGTAGGTTGTGCTGG-3' \\
\hline & $\operatorname{Rev}$ & 5'-ACAATGTTTCCCCAGAGAGCA-3' \\
\hline \multirow[t]{2}{*}{ SIRT1 } & Fwd & $5^{\prime}-\mathrm{ATCGGCTACCGAGACAAC}-3^{\prime}$ \\
\hline & $\operatorname{Rev}$ & 5'-GTCACTAGAGCTGGCGTGT-3' \\
\hline
\end{tabular}

CPT-1a, carnitine palmitoyltransferase $1 \alpha$; FGFR, fibroblast growth factor receptor; Fwd, forward; FXR, farnesoid x receptor; GAPDH, glyceraldehyde-3phosphate dehydrogenase; $\mathrm{LCN}$, lipocaline; MCP, monocyte chemoattractant protein; MIP, macrophage inflammatory protein; $\mathrm{PGC}$, peroxisome proliferatoractivated receptor $\gamma$ co-activator; PPAR, peroxisome proliferator-activated receptor; Rev, reverse; SAA, serum amyloid $A ; \beta-K L, \beta$-Klotho. 
threshold $\left(\Delta \Delta \mathrm{C}_{\mathrm{t}}\right)$ method by normalizing target mRNA $\mathrm{C}_{\mathrm{t}}$ values to those for glyceraldehyde-3-phosphate dehydrogenase $\left(\Delta \mathrm{C}_{\mathrm{t}}\right)$ with the control group $\left(\Delta \Delta \mathrm{C}_{\mathrm{t}}\right)$.

\section{Western Blot Analyses}

Western blot analyses were performed using liver nuclear extracts or whole liver extracts. The preparation of nuclear and cytoplasmic extracts from cells or tissue was performed using Activ Motif's Nuclear Extract Kit (Carlsbad, CA). Nuclear and cell extracts were then separated by electrophoresis in 10\% and/or AnyKd Mini-Protean TGX gels using Bio-Rad Mini-PROTEAN Tetra Cell System and transferred to polyvinylidene difluoride membranes via BioRad Blotting Module. Multiple protein band quantification and analysis were performed using AlphaView software version 3.4 (ProteinSimple, San Jose, CA).

\section{Studies with Mouse AML-12 Cell Hepatocytes}

Mouse AML-12 hepatocytes (ATCC, Manassas, VA) were cultured in Dulbecco's modified Eagle's medium/F12 medium supplemented with $10 \%$ fetal bovine serum, 100 $\mu \mathrm{g} / \mathrm{mL}$ streptomysin, $63 \mu \mathrm{g} / \mathrm{mL}$ penicillin $\mathrm{G}, 0.1 \mu \mathrm{mol} / \mathrm{L}$ dexamethasone, and insulin-transferrin-selenium (GibcoBRL). ${ }^{19,23}$ For the treatment, hepatocytes were grown at $37^{\circ} \mathrm{C}$ in an atmosphere of $5 \% \mathrm{CO}_{2}$. At approximately $80 \%$ confluence, the cells were then incubated for 16 hours in serum-free medium. Ethanol $(50 \mathrm{mmol} / \mathrm{L})$ or LPS (100 $\mathrm{ng} /$ $\mathrm{mL})$ was added for 24 hours.

\section{Measurement of Intracellular TG}

Cellular triglycerides (TGs) in the total lipid fraction were determined using a commercially available enzymatic kit (Cayman Chemical) or Nile Red Staining assays. ${ }^{18,19}$

Transfection, Luciferase Reporter Assay, and Adenovirus (Ad) Infection Studies in Mouse AML-12 Hepatocytes

Transfection studies were performed using Lipofectamine 2000 Reagent (Invitrogen) as described previously. ${ }^{24}$ For pGL3-24p3/LCN2-Luciferase reporter assays, plasmids were transiently transfected into AML-12 hepatocytes by Lipofectamine reagent for 48 hours. Luciferase assays were performed with cell extracts, and the results were averaged to represent a single data point for each transfection. $\beta$ Galactosidase ( $\beta$-Gal) was used as an internal control to correct for transfection efficiency. The replication deficient Ads were amplified in human embryonic kidney 293 cells and purified by Adenopure kits. Mouse AML-12 hepatocytes were infected with Ads for 36 to 48 hours before the experiments.
Human Liver Samples from Patients with AFLD and Normal Human Livers

Normal human livers and AFLD samples were obtained from the Liver Tissue Procurement and Distribution System, University of Minnesota. ${ }^{25}$ The inclusion criteria have been previously described. ${ }^{25}$ AFLD samples were collected from donor livers that were not used for transplantation because of excessive steatosis from the Medical College of Virginia through the Liver Tissue Procurement and Distribution System. AFLD was diagnosed on the basis of the history of alcohol drinking and liver histology. Normal healthy liver samples were collected from the part of donor livers that was not used for transplantation through the Liver Tissue Procurement and Distribution System.

\section{Statistical Analysis}

Statistical analysis was performed with Prism software version 6 (GraphPad Software, La Jolla, CA). Data are presented as means \pm SEM. For human liver samples, differences between groups were analyzed using a two-tailed unpaired Student's $t$-test (for human liver samples), with $P<0.05$ considered statistically significant. For comparison of multiple groups, one-way analysis of variance test followed by the Newman-Keuls multiple ranges test, with a statistically significant difference defined as $P<0.05$.

\section{Results}

\section{LCN2 Regulates Ethanol-Induced Cellular Lipid Accumulation in Mouse AML-12 Hepatocytes}

Mouse AML-12 hepatocytes efficiently metabolize ethanol and display many characteristics similar to isolated primary hepatocytes and provide an adequate in vitro alcoholic steatosis model. ${ }^{24}$ Recombinant LCN2 induced a significant, concentration-dependent increase in the TGs contents of AML-12 hepatocytes (Figure 1A and Supplemental Figure S1A). More important, ethanol exposure augmented recombinant LCN2-induced lipid accumulation in AML-12 cells (Supplemental Figure S1A). Compared with the adenovirus (Ad)-GFP control, Ad-LCN2 infection to AML12 cells significantly increased cellular fat accumulation, whereas knocking down LCN2 by Ad-LCN2siRNA did not alter cellular lipid contents (Figure 1B). Although ethanol significantly exacerbated cellular fat accumulation in Ad-LCN2-infected AML-12 cells, reduction of LCN2 levels by infecting Ad-LCN2siRNA blocked the ability of ethanol to induce fat accumulation (Figure 1B).

Ethanol consumption is correlated with elevated LPS level, which can promote the cellular lipid accumulation in hepatocytes (data not shown). We further used combination of ethanol and LPS $(\mathrm{E}+\mathrm{L})$ to examine the role of LCN2 in ethanol-induced cellular steatosis. Although $\mathrm{E}+\mathrm{L}$ significantly induced fat accumulation, Ad-LCN2 exacerbated 
A

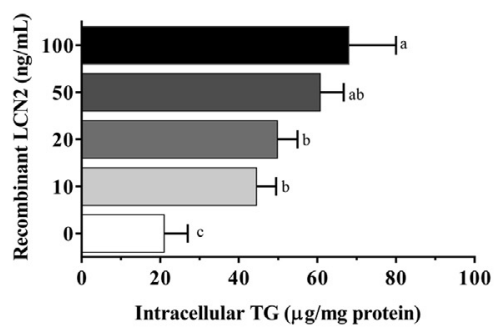

C

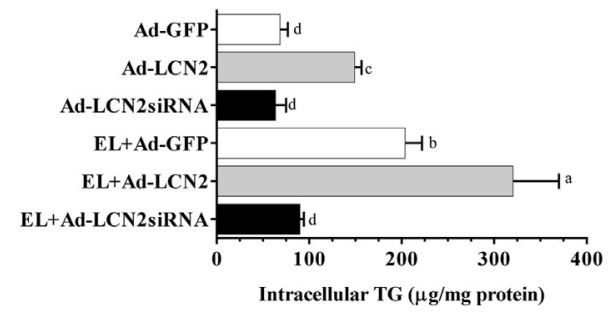

B
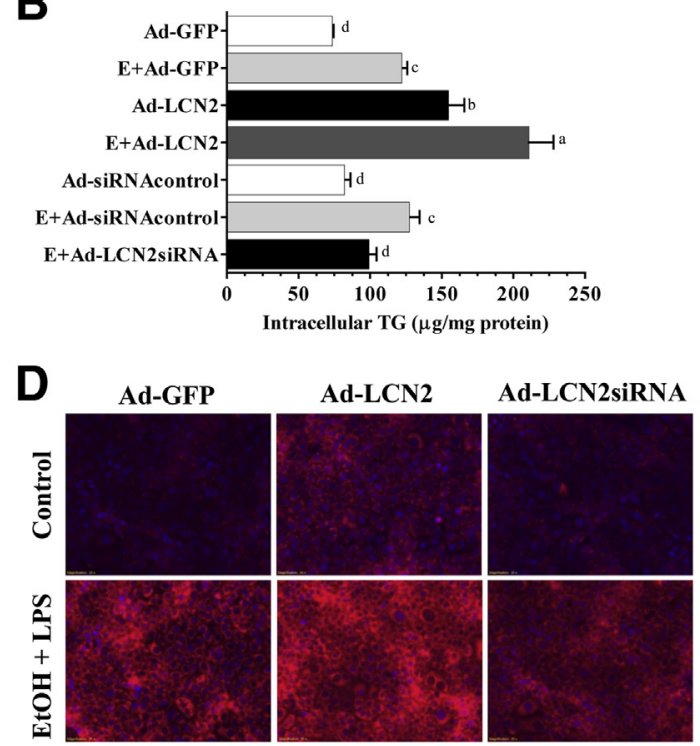

Figure 1 Lipocaline-2 (LCN2) regulates ethanol-induced cellular lipid accumulation in mouse AML-12 hepatocytes. A: AML-12 cells were cultured in media containing various concentrations of recombinant LCN2 as indicated for 48 hours. The cellular triglyceride (TG) content was measured using a triglyceride kit and normalized to the total protein content. B: AML-12 cells were infected with Ad-green fluorescent protein (GFP), Ad-LCN2, Ad-siRNAcontrol, or AdLCN2siRNA. Forty-eight hours after transfection, $50 \mathrm{mmol} / \mathrm{L}$ ethanol (EtOH; E) was added. After incubation for 24 hours, cellular TG contents were measured by a cellular enzymatic kit. C: AML-12 cells were infected with Ad-GFP, Ad-LCN2, Ad-siRNAcontrol, or Ad-LCN2siRNA for 48 hours, 50 mmol/L E and $100 \mathrm{ng} / \mathrm{mL}$ lipopolysaccharide (LPS; L) (E + L) were added for 24 hours. Cellular TG contents were measured by a cellular enzymatic kit. D: AML-12 cells were treated as in Figure 1C, cellular TG contents were visualized by Nile Red staining. Data are means \pm SEM from three to five experiments (A-C). Means without a common letter are significantly different, $P<0.05(\mathbf{A}-\mathbf{C})$.

$\mathrm{E}+$ L-mediated lipid accumulation in AML-12 hepatocytes (Figure 1, C and D). Remarkably, knocking down LCN2 in those cells completely prevented the fat accumulation effects of $\mathrm{E}+\mathrm{L}$ (Figure 1, C and D). Collectively, those results clearly demonstrated that LCN2 played a pivotal and causal role in lipid accumulation in AML-12 hepatocytes.

\section{Deletion of LCN2 Partially Ameliorates Ethanol- Induced Fatty Liver Injury in Mice}

We investigated in vivo function of LCN2 in the development of alcoholic fatty liver injury by 4 -week pair-feeding WT and LCN2 knockout (Lcn2KO) mice with control or ethanol-containing Lieber-DeCarli diets. ${ }^{19,22,24,25}$ Lcn2KO mice were on the C57BL/6 background and phenotypically normal under a chow diet. ${ }^{13}$

Ethanol feeding to WT mice significantly increased the levels of LCN2 mRNA expression to approximately $145 \%$ of WT controls and serum LCN2 levels to approximately $200 \%$ of WT controls (Supplemental Figure S1B and Figure 2A), respectively. LCN2 were completely removed in the $L c n 2 \mathrm{KO}$ mice fed with or without ethanol (Figure 2A and Supplemental Figure S1B). Compared to the WT controls, hepatic expression of 24p3R mRNA was significantly lower in the ethanol-fed WT mice, but markedly increased in the control-fed Lcn $2 \mathrm{KO}$ mice, and partially restored in the ethanol-fed Lcn2KO mice (Supplemental Figure S1B).

Liver TG level in the ethanol-fed WT mice was approximately threefold of the pair-fed WT control mice (Figure 2B).
Hepatic TGs were similar in the control diet pair-fed WT mice and Lcn2KO mice. In comparison with the ethanol-fed WT mice, the amount of hepatic TGs in the ethanol-fed Lcn $2 \mathrm{KO}$ mice was significantly lower by approximately $25 \%$ (Figure 2B). Serum ALT levels were not significantly altered in the ethanol-fed WT mice compared to the controlfed WT mice (Figure 2C). However, serum ALT was significantly lower in the Lcn2KO mice fed either with or without ethanol compared to the WT controls (Figure 2C). Compared to the WT controls, plasma AST was significantly elevated in the ethanol-fed WT mice, but normalized in the ethanol-fed Lcn2KO mice (Figure 2D). Concordantly, histological analysis revealed abundant accumulation of lipid droplets in the livers of ethanol-fed WT mice, but rare in the livers of WT control mice and of Lcn2KO mice fed either with or without ethanol (Figure 2E).

Immunohistochemically staining for $\mathrm{F} 4 / 80^{+}$(a marker for macrophage activation) and MPO (a marker for neutrophil infiltration) further revealed a higher level of those indicators of inflammation in the livers of ethanol-fed WT mice compared to the livers of WT controls (Figure 2, F and G). Strikingly, Lcn2KO mice fed either with or without ethanol displayed attenuated staining of liver $\mathrm{F} 4 / 80^{+}$or MPO compared with the ethanol-fed WT mice (Figure 2, F and G).

To further investigate the effects of LCN2, hepatocytespecific LCN2 knockout (Lcn2LKO) and WT mice were administrated with ethanol for 4 weeks using the same Lieber-DeCarli ethanol feeding protocol. ${ }^{19,22,24,25}$ Surprisingly, ablation of hepatocyte-specific LCN2 did not prevent 
A

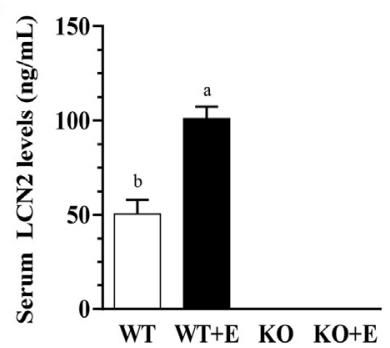

$\mathbf{E}$

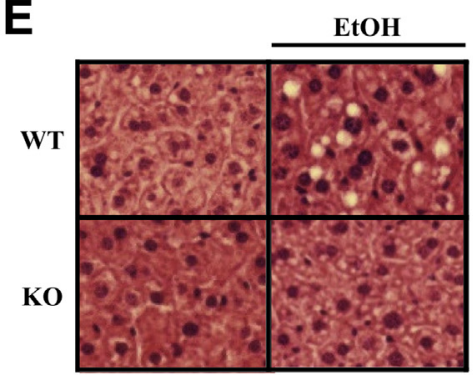

B

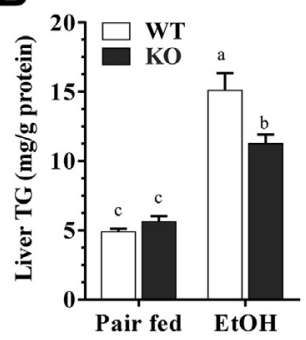

$\mathbf{F}$

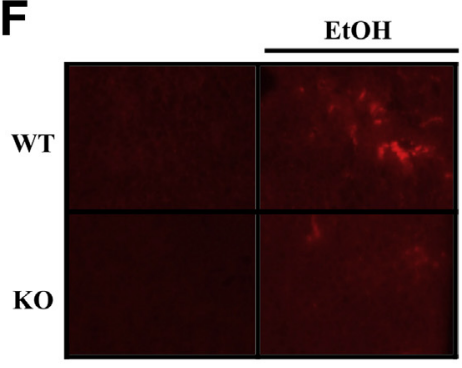

D
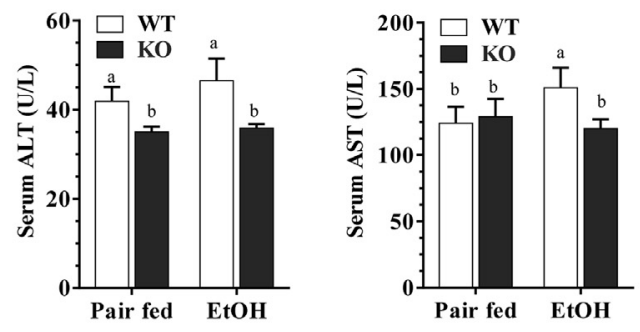

G

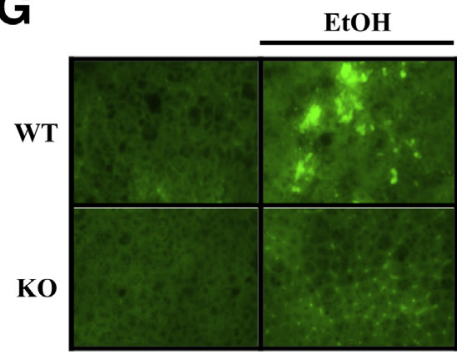

Figure 2 Global ablation of lipocaline-2 (LCN2) partially alleviates alcoholic fatty liver injury in mice. Wild-type (WT) or LCn2K0 (K0) mice were pair fed either a control diet or an ethanol-containing (EtOH; E) diet for 4 weeks. A: Serum LCN2 protein levels. B: Hepatic triglyceride (TG) contents. C: Serum alanine aminotransferase (ALT) levels. D: Serum aspartate aminotransferase (AST) levels. E: Hematoxylin and eosin staining of liver sections. F: Immunohistochemical staining of liver sections for $\mathrm{F} 4 / 80^{+}$. G: Immunohistochemical staining of liver sections for myeloperoxidase. Results are expressed as means \pm SEM (A-D). $n=4$ to 11 mice $(\mathbf{A}-\mathbf{D})$. Means without a common letter are significantly different, $P<0.05$ (A-D). Original magnification, $\times 40(\mathbf{E}-\mathbf{G})$.

the development of alcohol-induced fatty liver injury in mice (Supplemental Figure S2, A-C).

Collectively, our results demonstrated that global ablation of LCN2 partially but significantly ameliorated ethanolinduced fatty liver injury in mice.

\section{Removal of LCN2 Attenuates Hepatic Inflammatory Response but Selectively Increases Proinflammatory Cytokines in the Ethanol-Fed Mice}

The mRNA levels of hepatic serum amyloid A-1 (SAA-1), a proinflammatory acute-phase protein, were substantially elevated in the ethanol-fed WT mice compared with the pair-fed WT controls (Figure 3), consistent with our earlier reports. ${ }^{18,19}$ However, removal of LCN2 largely abolished the elevation of SAA-1 mRNA levels in the livers of Lcn2KO mice fed either with or without ethanol (Figure 3).

Serum levels of SAA-1 were significantly elevated in the ethanol-fed WT mice to approximately $152 \%$ and in the ethanol-fed $L c n 2 \mathrm{KO}$ to approximately $127 \%$, respectively, compared to the WT controls (Supplemental Figure S2D). However, in comparison with the ethanol-fed WT mice, circulating SAA-1 were reduced in the ethanol-fed Lcn $2 \mathrm{KO}$ mice by approximately $9 \%$ without statistical significance (Supplemental Figure S2D).

Although the mRNA levels of tumor necrosis factor- $\alpha$, macrophage inflammatory protein $-1 \alpha$, and $\mathrm{F} 4 / 80^{+}$were unchanged between the ethanol-fed WT and WT controls, mRNAs of those inflammatory cytokines were slightly but significantly reduced in the Lcn2 $\mathrm{KO}$ mice fed either with or without ethanol (Figure 3). On the contrary, hepatic mRNA levels of IL-6 were significantly increased in the ethanol-fed WT mice compared to the WT controls, and removal of LCN2 greatly augmented the increases in IL6 gene expression in the control-fed Lcn $2 \mathrm{KO}$ mice and to a lesser extent in the ethanol-fed $L c n 2 \mathrm{KO}$ mice. Furthermore, the mRNAs of IL-1 $\beta$ and monocyte chemoattractant protein-1 were also significantly elevated in the ethanol-fed $L c n 2 \mathrm{KO}$ mice compared to all other groups (Figure 3).

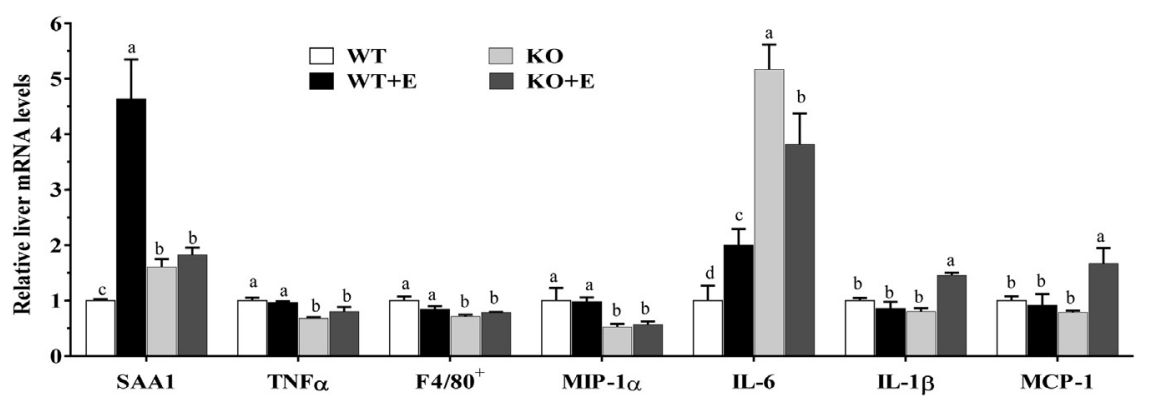

Figure 3 Lipocaline-2 (LCN2) deficiency attenuates ethanol-induced hepatic inflammation in mice. Wild-type (WT) or LCn2KO (KO) mice were pair fed either a control diet or an ethanol (E)containing diet for 4 weeks. Relative mRNA expression levels of hepatic serum amyloid $A 1$ (SAA1), tumor necrosis factor (TNF) $\alpha, \mathrm{F} 4 / 80^{+}$, macrophage inflammatory protein (MIP)- $1 \alpha$, IL-6, $\mathrm{IL}-1 \beta$, and monocyte chemoattractant protein (MCP)-1. Results are expressed as means \pm SEM. $n=4$ to 11 mice. Means without a common letter are significantly different, $P<0.05$. 
Taken together, these observations demonstrated that LCN2 deficiency selectively attenuated gene expression of hepatic inflammatory molecules, such as SAA-1, but upregulated certain proinflammatory cytokines in mice after ethanol administration.

\section{Deletion of LCN2 Stimulates Hepatic Nampt-SIRT1 Axis in Mice}

To dissect the molecular mechanisms by which LCN2 deficiency ameliorated alcoholic fatty liver injury, we examined the effects of LCN2 on hepatic sirtuin 1 (SIRT1) signaling both in vitro and in vivo. ${ }^{26}$

SIRT1 protein expression levels were significantly reduced by recombinant LCN2 in a dose-dependent manner in the AML-12 hepatocytes (Supplemental Figure S3A). More important, ectopic expression of SIRT1wt in AML-12 hepatocytes abolished the ability of recombinant LCN2 to induce fat accumulation, but inhibition of SIRT1 by SIRT1siRNA markedly exacerbated TG induction by LCN2, indicating that LCN2 induced lipid accumulation by perturbing SIRT1 activity (Supplemental Figure S3B). Note that expression or inhibition of SIRT1 by infecting the Ad-SIRT1wt or Ad-SIRT1siRNA was demonstrated via Western blot analysis (Supplemental Figure S3C).

Ethanol feeding significantly reduced the mRNA and protein levels of hepatic SIRT1 in WT mice compared to the control-fed WT mice (Figure 4, A-C). Removal of LCN2 significantly increased the SIRT1 mRNA and protein in the Lcn $2 \mathrm{KO}$ mice fed either with or without ethanol to levels significantly higher than that in the WT control mice (Figure 4, A-C).

Nicotinamide phosphoribosyltransferase (Nampt) regulates SIRT1 activity. ${ }^{27}$ Although ethanol feeding to WT mice inhibited mRNA and protein expression of Nampt compared to the control-fed WT mice, Nampt expression in the ethanol-fed Lcn $2 \mathrm{KO}$ mice was restored to the WT control levels (Figure 4, A-C).

Collectively, LCN2 deficiency ameliorated alcoholic fatty liver injury in mice in part by stimulating a central hepatic signaling, Nampt-SIRT1 axis.

\section{Removal of LCN2 Ameliorates Ethanol-Mediated Abnormalities in Hepatic Lipid Oxidation and Alleviates Ethanol-Caused Aberrant Hepatic Fibroblast Growth Factor 15/19 Signaling in Mice}

The mRNA expression of several molecules involved in hepatic fatty acid oxidation, including carnitine palmitoyltransferase $1 \alpha$, peroxisome proliferator-activated receptor $\gamma$ co-activator $1 \alpha$, and peroxisome proliferator-activated receptor $\alpha$, were not significantly altered in the ethanol-fed WT mice and in the control-fed Lcn2KO mice, but were elevated significantly in the ethanol-fed Lcn2KO mice, compared to the WT control mice (Figure 4D). LCN2 deficiency had no obvious effect on hepatic sterol regulatory

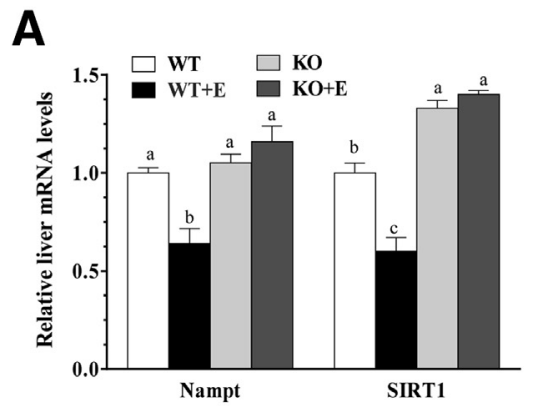

D

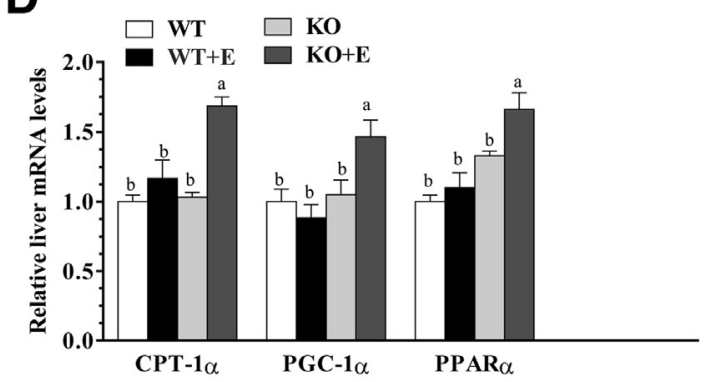

B

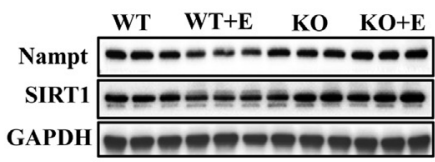

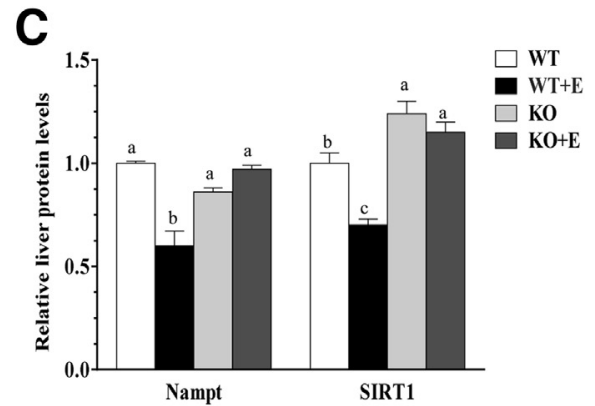

E

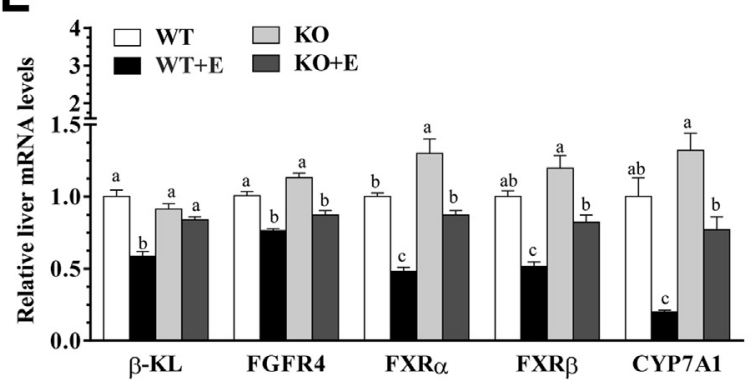

Figure 4 Ablation of lipocaline-2 (LCN2) activates the hepatic nicotinamide phosphoribosyltransferase (Nampt)-SIRT1 axis in ethanol-fed mice. Wild-type (WT) or Lcn2KO (KO) mice were pair fed either a control diet or an ethanol-containing diet for 4 weeks. A: Relative mRNA levels of hepatic Nampt and SIRT1. B: Representative Western analysis of hepatic Nampt or SIRT1 protein expression levels. C: Relative liver protein levels of Nampt and SIRT1. D: Relative mRNA levels of hepatic carnitine palmitoyltransferase $1 \alpha$ (CPT1 $\alpha)$, MCAD, peroxisome proliferator-activated receptor $\gamma$ co-activator $1 \alpha$ (PGC-1 $\alpha$ ), and peroxisome proliferator-activated receptor $\alpha$ (PPAR $\alpha$ ). E: Relative mRNA levels of hepatic $\beta$-Klotho ( $\beta$-KL), farnesoid $x$ receptor (FXR) $\alpha$, FXR $\beta$, fibroblast growth factor receptor (FGFR4), and CYP7A1. Results are expressed as means \pm SEM (three to five in vitro experiments) $(\mathbf{A}, \mathbf{C}-\mathbf{E})$. $n=4$ to 11 mice $(\mathbf{A}, \mathbf{C}-\mathbf{E})$. Means without a common letter are significantly different, $P<0.05(\mathbf{A}, \mathbf{C}-\mathbf{E})$. 
element binding protein-1-mediated lipogenesis in mice after ethanol administration (data not shown).

Gut-secreted fibroblast growth factor (FGF) 15 (human FGF19) plays a vital role in regulating hepatic lipid metabolism and bile acid synthesis via its hepatic receptors, fibroblast growth factor receptor 4 (FGFR4)/ $\beta$-Klotho $(\beta-K L) .{ }^{28}$ We investigated whether hepatic FGF15/19 signaling might play a role in the prevention of alcoholic fatty liver by LCN2 deficiency in mice. In comparison with the WT controls, mRNA expression of liver $\beta$-KL was significantly lower in the ethanol-fed WT mice (Figure 4E). Removal of LCN2 significantly reduced the suppression of hepatic $\beta$-KL mRNA on ethanol feeding (Figure 4E). Interestingly, ethanol feeding inhibited FGFR4 mRNAs to similar extent in both the WT mice and Lcn $2 \mathrm{KO}$ mice (Figure 4E). The mRNA levels of farnesoid $\mathrm{x}$ receptor (FXR) $\alpha, \quad$ FXR $\beta$, and cholesterol $7 \alpha$-hydroxylase (CYP7A1), three known molecules involved in mediating hepatic FGF15/19 signaling ${ }^{28}$ were significantly inhibited in the ethanol-fed WT mice in comparison with the WT controls. However, the mRNA levels of FXR $\alpha, \operatorname{FXR} \beta$, and CYP7A1 in the ethanol-fed Lcn $2 \mathrm{KO}$ mice were restored to their WT control levels (Figure 4E).

Taken together, these data suggested that removal of LCN2 in mice ameliorated the ethanol-mediated abnormalities in hepatic lipid oxidation, and alleviated the ethanol-induced aberrant hepatic FGF15/19 signaling.

\section{Deletion of LCN2 Activates Chaperone-Mediated Autophagy in the Livers of Ethanol-Fed Mice}

Ethanol feeding to WT mice significantly inhibited the chaperone-mediated autophagy activity with decreased mRNA expression levels of heat shock protein (Hsp) a8, Hsp90aal, and Hsp70, and lysosome-associated membrane protein type 2A (LAMP-2A) compared to the WT controls (Figure 5A). ${ }^{29,30}$ Remarkably, mRNA expression levels of
Hspa8, Hsp90aa1, Hsp70, in ethanol-fed Lcn2KO mice were restored to their WT control levels, whereas LAMP2A mRNA was also partially restored to the WT controls (Figure 5A).

We further analyzed two major markers of autophagic flux: microtubule-associated protein 2 light chain 3 (LC3)-II and p62/sequestosome 1 (SQSTM1) (p62). ${ }^{31}$ In comparison with the WT control mice, the protein levels of LC3-II were significantly increased by ethanol feeding to the WT mice (Figure 5B). However, LCN2 deficiency significantly reduced the LC3-II protein expression, and this reduction was more pronounced in the livers of ethanol-fed Lcn2KO mice (Figure 5, B and C). The protein expression levels of P62 were significantly reduced in both ethanol-fed WT mice and control-fed Lcn $2 \mathrm{KO}$ compared to the WT controls. However, the protein levels of P62 were further suppressed in the livers of ethanol-fed $L c n 2 \mathrm{KO}$ mice (Figure 5, B and D). Note that chronic ethanol feeding significantly inhibited mRNA expression levels of p62 and LC3 in both WT and Lcn2KO mice (Supplemental Figure S4A).

Taken together, our results demonstrated that removal of LCN2 selectively activated hepatic chaperone-mediated autophagy in mice after ethanol administration.

\section{Up-Regulation of LCN2 in Response to Ethanol Exposure in Mouse AML-12 Hepatocytes}

The LCN2 reporter activity and mRNA and protein levels of LCN2 are significantly increased by incubation with either ethanol or LPS in a concentration-dependent manner in AML-12 hepatocytes (Supplemental Figure S5). These effects of ethanol and LPS on the reporter activity and mRNA levels of LCN2 are accumulative (Supplemental Figure S5), demonstrating the ability of ethanol, LPS, or $\mathrm{E}+\mathrm{L}$ to directly induce $\mathrm{LCN} 2$ expression in hepatocytes.

Although ethanol significantly induced LCN2 mRNA expression, $0.1 \mathrm{mmol} / \mathrm{L} \mathrm{ADH}$ inhibitor 4-methylpyrazole (4-MP) significantly augmented the ethanol-mediated
A
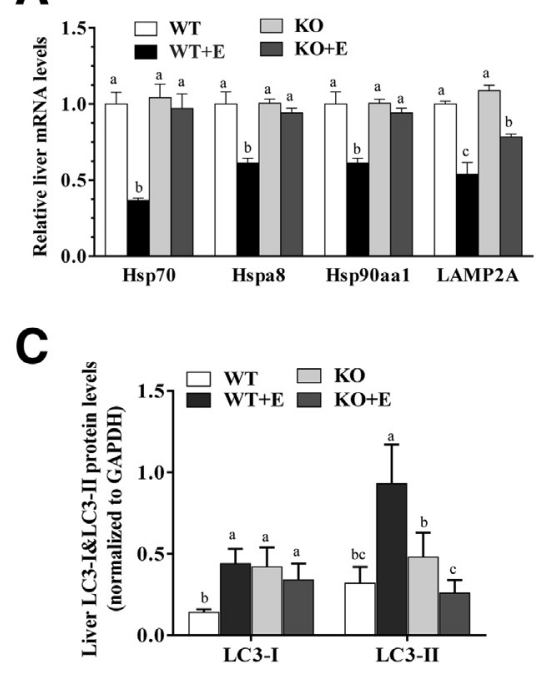

B

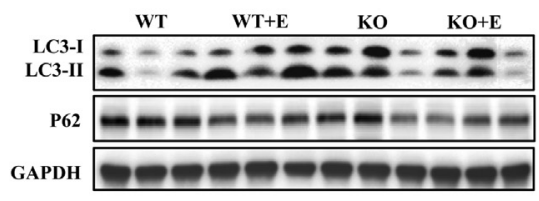

D

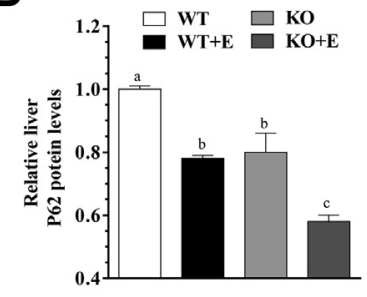

Figure 5 Removal of lipocaline-2 (LCN2) stimulates hepatic chaperone-mediated autophagy in ethanol-fed mice. Wild-type (WT) or Lcn2KO (KO) mice were pair fed either a control diet or an ethanol (E)-containing diet for 4 weeks. A: Relative mRNA levels of hepatic Hspa8, Hap90aa1, Hsp70, and LAMP2A. B: Representative Western blot analysis of hepatic LC3-I, IC3-II, and p62 protein expression levels. C: Protein levels of hepatic LC3-I or LC3-II. D: Relative liver p62 protein levels. Results are expressed as means \pm SEM (A, C, and D). $n=4$ to 11 mice (A, C, and D). Means without a common letter are significantly different, $P<0.05$ (A, C, and D). 
increases in LCN2 gene expression in AML-12 cells; 0.1 $\mathrm{mmol} / \mathrm{L}$ ALDH2 inhibitor cyanamide largely abolished the ability of ethanol to induce LCN2, suggesting the involvement of acetaldehyde but not acetate in the up-regulation of LCN2 (Figure 6A).

Furthermore, MG 132 (a known proteasome inhibitor, and also an inhibitor of NF- $\mathrm{KB}$ signaling) or cyclosporine A [a known nuclear factor of activated T cells c4 (NFATc4) inhibitor] completely abolished the ethanol-mediated increases in LCN2 mRNA levels in AML-12 cells, indicating the involvement of both NF- $\kappa \mathrm{B}$ and NFATc4 in the ethanolmediated up-regulation of LCN2 (Figure 6B). MG132 and cyclosporine A are chemical inhibitors and exhibit nonspecific or off-target effects.

\section{Ethanol-Mediated Perturbation of miR-217-SIRT1 Axis Induces LCN2 in AML-12 Hepatocytes}

Overexpression of miR-217 in AML-12 hepatocytes markedly increased the LCN2 mRNA levels and miR-217 exacerbated the ethanol-mediated up-regulation of LCN2 (Figure 6C). ${ }^{32}$ Conversely, miR-217 inhibition by anti-miR217 or overexpression of SIRT1wt in hepatocytes completely abolished the ability of ethanol to induce LCN2, suggesting a causal role of miR-217-SIRT1 axis in mediating the effect of ethanol on LCN2 (Figure 6, C and D). Consistent with these in vitro findings, LCN2 protein levels were substantially increased in the serum samples from the previously studied ethanol-fed WT mice, and further significantly augmented in the ethanol-fed liver-specific deletion of Sirt1 (Sirt1LKO) mice (Supplemental Figure S6). ${ }^{18}$

Taken together, these results suggested that ethanolmediated perturbation of miR-217-SIRT1 axis led to
LCN2 induction in hepatocytes. Given that LCN2 inhibits SIRT1, miR-217-SIRT1 axis may constitute a positive regulatory loop in the up-regulation of $\mathrm{LCN} 2$ by ethanol.

\section{Dysregulation of LCN2 and Several LCN2-Regulated Molecules in Patients with AFLD}

To establish clinical relevance of LCN2 induction in the pathogenesis of AFLD, we examined mRNA expression levels of LCN2 and several molecules regulated by LCN2 in the liver samples from patients with AFLD. ${ }^{25}$ LCN2 mRNA levels showed a tendency of elevation (without reaching statistical significance) in the alcoholic fatty liver disease samples compared to the normal livers (Figure 7). In comparison with healthy human livers, AFLD samples had significantly lower $\beta$-KL and CYP7A1 mRNA levels (Figure 7). Moreover, the mRNAs of LAMP2A, Hsp70, and Hsp90aal were significantly lower in patients with AFLD compared to the normal controls (Figure 7).

Collectively, those data suggested that dysregulation of LCN2 expression and impairment of LCN2-mediated molecules might contribute, at least in part, to the development and progression of human ALD.

\section{Discussion}

In the present study, we identified a pivotal and multifunctional role of LCN2 in the early stage of alcoholic liver disease, AFLD. In cultured AML-12 hepatocytes, treatment of recombinant LCN2 or infection of Ad-LCN2 significantly exacerbated ethanol-induced cellular TG accumulation. Remarkably, knocking down LCN2 in hepatocytes by infecting Ad-LCN2siRNA completely prevented the fat
A
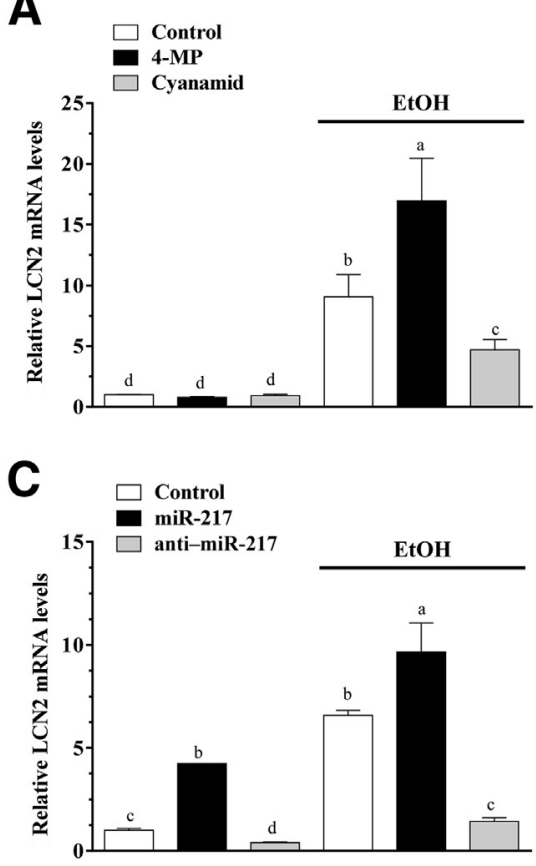

B

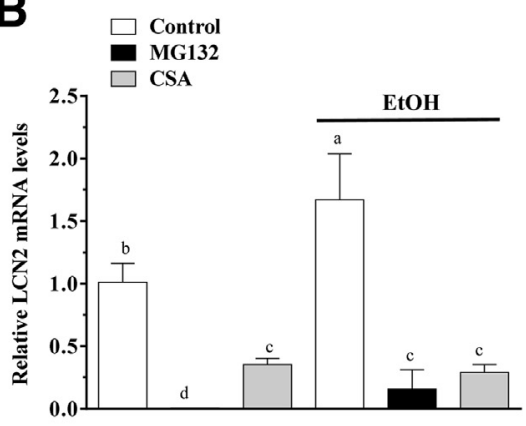

D

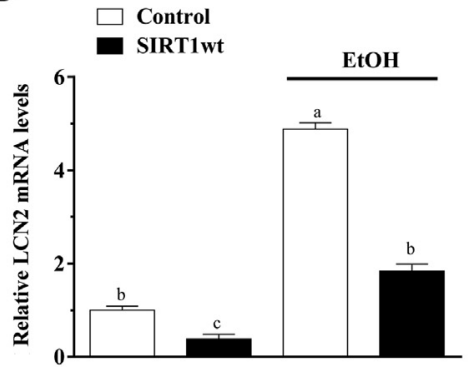

Figure 6 Up-regulation of lipocaline-2 (LCN2) in response to ethanol (EtOH) exposure in mouse AML-12 hepatocytes. A: AML-12 cells were cultured in media containing $0.1 \mathrm{mmol} / \mathrm{L}$ 4-methylpyrazole (4-MP) or $0.1 \mathrm{mmol} / \mathrm{L}$ cyanamid in the presence or absence of $50 \mathrm{mmol} / \mathrm{L}$ ethanol for 24 hours. Quantitative RT-PCR (RT-qPCR) was used to estimate relative mRNA levels of LCN2. B: AML-12 cells were cultured in media containing $40 \mu \mathrm{mol} / \mathrm{L}$ MG132 or $5 \mu \mathrm{g} / \mathrm{mL}$ cyclosporine A (CSA) in the presence or absence of $50 \mathrm{mmol} / \mathrm{L}$ ethanol for 24 hours. RT-qPCR was used to estimate relative mRNA levels of LCN2. C: AML-12 cells were transfected with control vector, miR-217, or anti-miR-217. Forty-eight hours after transfection, $50 \mathrm{mmol} / \mathrm{L}$ ethanol was added. After incubation for 24 hours, RT-qPCR was used to estimate relative mRNA levels of LCN2. D: AML-12 cells were transfected with control vector, or SIRT1wt. Forty-eight hours after transfection, $50 \mathrm{mmol} / \mathrm{L}$ ethanol was added. After incubation for 24 hours, RT-qPCR was used to estimate relative mRNA levels of LCN2. All data are given as means \pm SEM from at least three to five experiments (A-D). Means without a common letter are significantly different, $P<0.05$. 


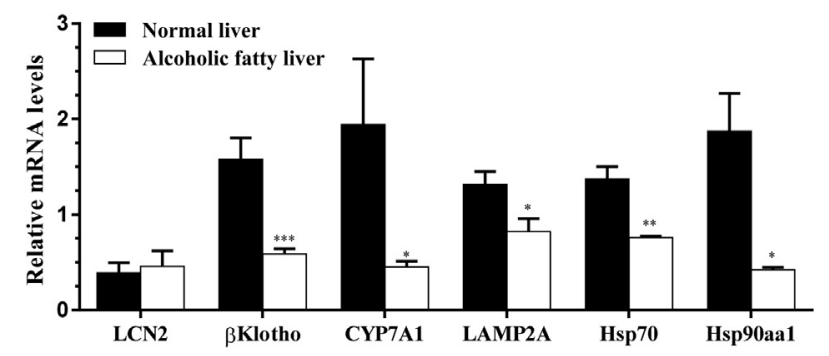

Figure 7 Hepatic mRNA levels of lipocaline-2 (LCN2) from patients with alcoholic fatty liver disease. Real-time PCR analyses of hepatic mRNA levels of LCN2, $\beta-K L$, CYP7A1, LAMP2A, Hsp70, and Hsp90 in normal human livers and livers from patients with alcoholic fatty liver. All data are expressed as means \pm SEM. $n=10$ (normal liver); $n=10$ (livers from patients with alcoholic fatty liver). ${ }^{*} P<0.05,{ }^{* *} P<0.01$, and ${ }^{* * *} P<0.001$ compared to normal livers.

accumulation induced by ethanol, indicating a causal role of LCN2 in a cellular model of alcoholic steatosis. Consistent with these observations, ablation of LCN2 partially but significantly alleviated alcoholic steatohepatitis in mice. Mechanistic studies revealed that the protective effects of LCN2 deficiency against alcoholic fatty liver injury was accompanied by stimulated hepatic Nampt-SIRT1 axis, restored endocrine metabolic regulatory hepatic FGF15/19 signaling, and activated chaperone-mediated autophagy. More important, LCN2 expression and LCN2-regulated molecules were dysregulated in the livers from patients with AFLD. Finally, ethanol exposure significantly induced LCN2 promoter activity and increased LCN2 gene expression in AML-12 hepatocytes. However, inhibition of miR217 or overexpression of SIRT1 in AML-12 hepatocytes largely abolished the ability of ethanol to induce LCN2, suggesting that miR-217-SIRT1 axis mediated the effect of ethanol on LCN2. Altogether, our results provided strong evidences that abnormally elevated LCN2 in response to ethanol played a complex and causative role in the experimental alcoholic fatty liver injury in mice through disrupting multiple signaling pathways (Figure 8).

Our in vitro data unequivocally demonstrated that the ethanol-induced excessive cellular lipid accumulation was mediated through LCN2 induction. Paradoxically, LCN2 deficiency only partially ameliorated alcoholic hepatosteatosis in mice. We observed that the ethanoladministrated Lcn2KO mice displayed higher levels of several hepatic proinflammatory cytokines, such as IL-1 $\beta$, IL-6, and monocyte chemoattractant protein-1. These elevated cytokines could compromise the protective ability of LCN2 deficiency and contribute to development of steatosis in the ethanol-fed Lcn2KO mice. More surprisingly, we found that removal of LCN2 did not alter steatosis in mice when ethanol was administrated with a chronicbinge ethanol feeding protocol (referred as Gao-binge ethanol treatment model) (data not shown). ${ }^{18,25}$ Therefore, it is likely that LCN2 selectively involves in the development and progression of chronically ethanol-induced steatosis.

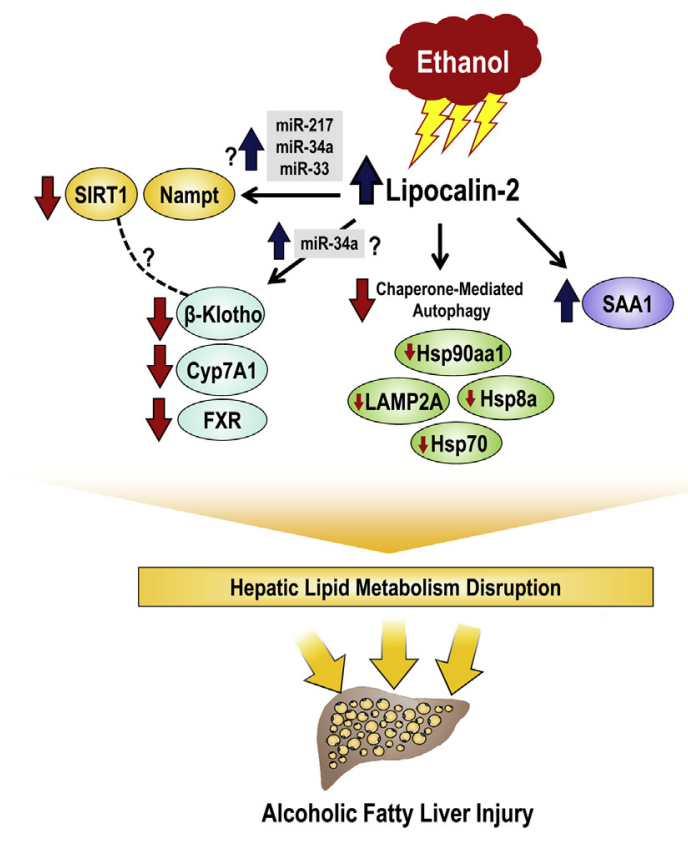

Figure 8 Proposed working model for the pivotal role played by lipocaline-2 (LCN2) in mediating detrimental effects of ethanol in the liver. Ethanol-mediated LCN2 induction is associated with disrupted hepatic nicotinamide phosphoribosyltransferase (Nampt)-SIRT1 axis, impaired endocrine metabolic regulatory hepatic fibroblast growth factor (FGF) 15/ 19 signaling, inhibited hepatic chaperone-mediated autophagy, and increased proinflammatory cytokine serum amyloid A-1 (SAA1) production leading to development of alcoholic fatty liver injury in mice.

The vitro data clearly demonstrate that hepatocytes were mainly responsible for LCN2 production after ethanol exposure. Indeed, in cultured RAW264.7 cells, exposure to ethanol inhibited the expression levels of LCN2 mRNA compared to controls, supporting that the ability of ethanol to directly induce LCN2 expression occurs in hepatocytes but not in macrophages (preliminary observations). Paradoxically, the protective phenotype observed in Lcn $2 \mathrm{KO}$ mice was not seen in hepatocyte-specific Lcn2LKO mice after ethanol administration. It is possible that the removal of hepatocyte-specific LCN2 is not sufficient to protect against the development of AFLD.

It is also intriguing that LCN2 mRNA levels only showed a tendency of elevation (did not reach statistical significance) in patients with AFLD compared to healthy humans. In addition to liver, LCN2 is highly expressed in various other tissues. ${ }^{2}$ LCN2 has been identified as a factor dramatically induced by dexamethasone and tumor necrosis factor- $\alpha$ in 3T3-L1 adipocytes and adipose tissue is a dominant site of LCN2 gene expression in mouse. ${ }^{33,34} \mathrm{We}$ recently observed that adipose LCN2 mRNA expression was drastically increased in mice after ethanol feeding, which, in turn, led to substantial increases in serum LCN2 levels (preliminary observations). Therefore, the ethanolmediated increases in circulating LCN2 might be also derived from adipose tissue, and subsequently contributed to the development of alcoholic fatty liver. How ethanol influences LCN2 induction in tissues such as adipose and its 
relationship with alcoholic fatty liver injury are currently under investigation in our laboratory.

The underlying mechanisms by which ethanol inhibits hepatic Nampt gene and protein expression in the ethanolfed mice remain unclear. The $3^{\prime}$ UTR of Nampt mRNA contains a functional binding site for miR-34a, and miR-34a reduces $\mathrm{NAD}^{+}$levels and SIRT1 activity by directly inhibiting Nampt. ${ }^{35}$ We have previously shown that hepatic miR-34a levels were significantly elevated in AML-12 hepatocytes exposed to ethanol or in livers of ethanol-fed mice. $^{32,36}$ Ethanol-mediated LCN2 may inhibit Nampt gene and protein expression via directly up-regulating miR34a levels. Intriguingly, similar to Nampt, miR-34a also selectively targets hepatic $\beta$-KL but not FGFR 4 by binding to the $3^{\prime} \mathrm{UTR}$ of $\beta-\mathrm{KL}$ mRNA. ${ }^{37}$ Therefore, it is logical to speculate that ethanol-mediated LCN2 induction may also down-regulate hepatic $\beta$-KL via up-regulating miR-34a.

Deficient chaperone-mediated autophagy in the liver leads to aberrant lipid metabolism and development of hepatosteatosis in mice. ${ }^{29,30}$ Ethanol-mediated induction of LCN2 specifically inhibits hepatic chaperone-mediated autophagy and may subsequently contribute to hepatic excessive fat accumulation in mice and in patients with alcoholic liver disease. Nevertheless, to establish a causal relationship, role of chaperone-mediated autophagy in the development of alcoholic fatty liver injury will need further investigation using genetically modified mouse models, such as liver-specific knockout mice for LAMP2A or transgenic mice overexpressing hepatic LAMP2A.

Regulation of iron metabolism represents an important mechanism of LCN2 action. ${ }^{2-4}$ Alcohol and iron have been shown to interact synergistically to cause liver injury. ${ }^{38}$ Paradoxically, ethanol-induced disruption of several molecules involved in the iron metabolism, such as ferritin, hepcidin, and iron, seems mediated via LCN2 independent manners (Supplemental Figure S4, B and C). Additional studies addressing the roles of LCN2-24p3R axis in regulating iron metabolism and its involvement in pathogenesis of alcoholic liver disease are needed.

In summary, we provide evidence to support the detrimental effects of ethanol-mediated LCN2 induction and its pathogenic implication in the initiation and progression of alcohol-induced fatty liver injury. In addition, we demonstrate, for the first time, that several novel mechanisms, including hepatic chaperone-mediated autophagy and endocrine metabolic regulatory FGF15/19 signaling, are involved in the development and progression of AFLD. Our findings suggest that targeting the LCN2 or modulating these hepatic machineries might provide new avenues for therapeutic intervention in human alcoholic liver disease.

\section{Acknowledgment}

We thank Dr. Tak Mak for providing global LCN2 knockout mice.

\section{Supplemental Data}

Supplemental material for this article can be found at http://dx.doi.org/10.1016/j.ajpath.2016.05.006.

\section{References}

1. Gao B, Bataller R: Alcoholic liver disease: pathogenesis and new therapeutic targets. Gastroenterology 2011, 141:1572-1585

2. Flower DR, North AC, Sansom CE: The lipocalin protein family: structural and sequence overview. Biochim Biophys Acta 2000, 1482: $9-24$

3. Goetz DH, Holmes MA, Borregaard N, Bluhm ME, Raymond KN, Strong RK: The neutrophil lipocalin NGAL is a bacteriostatic agent that interferes with siderophore-mediated iron acquisition. Mol Cell 2002, 10:1033-1043

4. Devireddy LR, Gazin C, Zhu X, Green MR: A cell-surface receptor for lipocalin $24 \mathrm{p} 3$ selectively mediates apoptosis and iron uptake. Cell 2005, 123:1293-1305

5. Makris K, Rizos D, Kafkas N, Haliassos A: Neurophil gelatinaseassociated lipocalin as a new biomarker in laboratory medicine. Clin Chem Lab Med 2012, 50:1519-1532

6. Zhao P, Elks CM, Stephens JM: The induction of lipocalin-2 protein expression in vivo and in vitro. J Biol Chem 2014, 289:5960-5969

7. Guo H, Bazuine M, Jin D, Huang MM, Cushman SW, Chen X: Evidence for the regulatory role of lipocalin 2 in high-fat diet-induced adipose tissue remodeling in male mice. Endocrinology 2013, 154: $3525-3538$

8. Guo H, Jin D, Zhang Y, Wright W, Bazuine M, Brockman DA, Bernlohr DA, Chen X: Lipocalin-2 deficiency impairs thermogenesis and potentiates diet-induced insulin resistance in mice. Diabetes 2010, 59:1376-1385

9. Borkham-Kamphorst E, Drews F, Weiskirchen R: Induction of lipocalin-2 expression in acute and chronic experimental liver injury moderated by pro-inflammatory cytokines interleukin- $1 \beta$ through nuclear factor- $\mathrm{\kappa B}$ activation. Liver Int 2011, 31:656-665

10. Borkham-Kamphorst E, van de Leur E, Zimmermann HW, Karlmark KR, Tihaa L, Haas U, Tacke F, Berger T, Mak TW, Weiskirchen R: Protective effects of lipocalin-2 (LCN2) in acute liver injury suggest a novel function in liver homeostasis. Biochim Biophys Acta 2013, 1832:660-673

11. Asimakopoulou A, Borkham-Kamphorst E, Henning M, Yagmur E, Gassler N, Liedtke C, Berger T, Mak TW, Weiskirchen R: Lipocalin-2 (LCN2) regulates PLIN5 expression and intracellular lipid droplet formation in the liver. Biochim Biophys Acta 2014, 1842:1513-1524

12. Lai HS, Wu YM, Lai SL, Lin WH: Lipocalin-2 gene expression during liver regeneration after partial hepatectomy in rats. Int J Surg 2013, 11: 314-318

13. Xu MJ, Feng D, Wu H, Wang H, Chan Y, Kolls J, Borregaard N, Porse B, Berger T, Mak TW, Cowland JB, Kong X, Gao B: Liver is the major source of elevated serum lipocalin-2 levels after bacterial infection or partial hepatectomy: a critical role for IL-6/STAT3. Hepatology 2015, 61:692-702

14. Wang Y, Lam KS, Kraegen EW, Sweeney G, Zhang J, Tso AW, Chow WS, Wat NM, Xu JY, Hoo RL, Xu A: Lipocalin-2 is an inflammatory marker closely associated with obesity, insulin resistance, and hyperglycemia in humans. Clin Chem 2007, 53:34-41

15. Semba T, Nishimura M, Nishimura $\mathrm{S}$, Ohara O, Ishige T, Ohno S, Nonaka K, Sogawa K, Satoh M, Sawai S, Matsushita K, Imazeki F, Yokosuka O, Nomura F: The FLS (fatty liver Shionogi) mouse reveals local expressions of lipocalin-2, CXCL1 and CXCL9 in the liver with non-alcoholic steatohepatitis. BMC Gastroenterol 2013, 13:120

16. Auguet T, Terra X, Quintero Y, Martínez S, Manresa N, Porras JA, Aguilar C, Orellana-Gavaldà JM, Hernández M, Sabench F, Lucas A, Pellitero S, Del Castillo D, Richart C: Liver lipocalin 2 expression in 
severely obese women with nonalcoholic fatty liver disease. Exp Clin Endocrinol Diabetes 2013, 121:119-124

17. Asimakopoulou A, Borkham-Kamphorst E, Tacke F, Weiskirchen R: Lipocalin-2 (NGAL/LCN2), a "help-me" signal in organ inflammation. Hepatology 2016, 63:669-671

18. Yin H, Hu M, Liang X, Ajmo JM, Li X, Bataller R, Odena G, Stevens SM Jr, You M: Deletion of SIRT1 from hepatocytes in mice disrupts lipin-1 signaling and aggravates alcoholic fatty liver. Gastroenterology 2014, 146:801-811

19. Hu M, Yin H, Mitra MS, Liang X, Ajmo JM, Nadra K, Chrast R, Finck BN, You M: Hepatic-specific lipin-1 deficiency exacerbates experimental alcohol-induced steatohepatitis in mice. Hepatology 2013, 58:1953-1963

20. Jiang Z, Zhou J, Zhou D, Zhu Z, Sun L, Nanji AA: The adiponectinSIRT1-AMPK pathway in alcoholic fatty liver disease in the rat. Alcohol Clin Exp Res 2015, 39:424-433

21. Wieser V, Tymoszuk P, Adolph TE, Grander C, Grabherr F, Enrich B, Pfister A, Lichtmanegger L, Gerner R, Drach M, Moser P, Zoller H, Weiss G, Moschen AR, Theurl I, Tilg H: Lipocalin 2 drives neutrophilic inflammation in alcoholic liver disease. J Hepatol 2016, 64: 872-880

22. Bertola A, Mathews S, Ki SH, Wang H, Gao B: Mouse model of chronic and binge ethanol feeding (the NIAAA model). Nat Protoc 2013, 8:627-637

23. Yu J, Chu ES, Wang R, Wang $\mathrm{S}$, Wu CW, Wong VW, Chan HL, Farrell GC, Sung JJ: Heme oxygenase-1 protects against steatohepatitis in both cultured hepatocytes and mice. Gastroenterology 2010, 138: 694-704

24. Hu M, Wang F, Li X, Rogers CQ, Liang X, Finck BN, Mitra MS, Zhang R, Mitchell DA, You M: Regulation of hepatic lipin-1 by ethanol: role of AMP-activated protein kinase/sterol regulatory element-binding protein 1 signaling in mice. Hepatology 2012, 55:437-446

25. Bertola A, Park O, Gao B: Chronic plus ethanol feeding synergistically induces neutrophil infiltration and liver injury in mice: a critical role for E-selectin. Hepatology 2013, 58:1814-1823

26. Shen Z, Liang X, Rogers CQ, Rideout D, You M: Involvement of mammalian sirtuin 1 in the action of ethanol in the liver. Am J Physiol Gastrointest Liver Physiol 2008, 294:G892-G898
27. Imai S, Yoshino J: The importance of NAMPT/NAD/SIRT1 in the systemic regulation of metabolism and ageing. Diabetes Obes Metab 2013, 15 Suppl 3:26-33

28. Cicione C, Degirolamo C, Moschetta A: Emerging role of fibroblast growth factors 15/19 and 21 as metabolic integrators in the liver Hepatology 2012, 56:2404-2411

29. Schneider JL, Suh Y, Cuervo AM: Deficient chaperone-mediated autophagy in liver leads to metabolic dysregulation. Cell Metab 2014, 20:417-432

30. Kaushik S, Cuervo AM: Chaperone-mediated autophagy: a unique way to enter the lysosome world. Trends Cell Biol 2012, 22:407-417

31. Lin CW, Zhang H, Li M, Xiong X, Chen X, Chen X, Dong XC, Yin XM: Pharmacological promotion of autophagy alleviates steatosis and injury in alcoholic and non-alcoholic fatty liver conditions in mice. J Hepatol 2013, 58:993-999

32. Yin H, Hu M, Zhang R, Shen Z, Flatow L, You M: MicroRNA-217 promotes ethanol-induced fat accumulation in hepatocytes by downregulating SIRT1. J Biol Chem 2012, 287:9817-9826

33. Yan QW, Yang Q, Mody N, Graham TE, Hsu CH, Xu Z, Houstis NE, Kahn BB, Rosen ED: The adipokine lipocalin 2 is regulated by obesity and promotes insulin resistance. Diabetes 2007, 56:2533-2540

34. Zhang J, Wu Y, Zhang Y, Leroith D, Bernlohr DA, Chen X: The role of lipocalin 2 in the regulation of inflammation in adipocytes and macrophages. Mol Endocrinol 2008, 22:1416-1426

35. Choi SE, Fu T, Seok S, Kim DH, Yu E, Lee KW, Kang Y, Li X, Kemper B, Kemper JK: Elevated microRNA-34a in obesity reduces $\mathrm{NAD}^{+}$levels and SIRT1 activity by directly targeting NAMPT. Aging Cell 2013, 12:1062-1072

36. Yin H, Liang X, Jogasuria A, Davidson NO, You M: miR-217 regulates ethanol-induced hepatic inflammation by disrupting sirtuin 1lipin-1 signaling. Am J Pathol 2015, 185:1286-1296

37. Fu T, Choi SE, Kim DH, Seok S, Suino-Powell KM, Xu HE, Kemper JK: Aberrantly elevated microRNA-34a in obesity attenuates hepatic responses to FGF19 by targeting a membrane coreceptor betaKlotho. Proc Natl Acad Sci U S A 2012, 109:16137-16142

38. Harrison-Findik DD: Is the iron regulatory hormone hepcidin a risk factor for alcoholic liver disease? World J Gastroenterol 2009, 15: $1186-1193$ 